\title{
Importance of nasal septal cartilage perichondrium for septum strength mechanics: a cadaveric study*
}

\author{
Nihal Seden Tekke1, Zeynep Alkan², Ozgur Yigit², Aylin Bekem³, \\ Ahmet Unal ${ }^{3}$, Feyzi Sahin ${ }^{4}$, Hasan Huseyin Balikci ${ }^{5}$, Engin Acioglu², \\ Rhinology 52: 167-171, 2014 \\ Yusuf Muhammed Durna ${ }^{2}$ \\ DOI:10.4193/Rhino13.199 \\ *Received for publication: \\ ' Department of ORL, Kahramanmaras Necip Fazıl State Hospital, Kahramanmaras, Turkey \\ November 18, 2013 \\ 2 Department of ORL, Istanbul Training and Research Hospital, Istanbul, Turkey \\ Accepted: January 5, 2014 \\ ${ }^{3}$ Department of Metallurgical and Materials Engineering, Faculty of Chemical Metallurgy, Yildiz Technical University, Istanbul, Turkey \\ ${ }^{4}$ Ministry of Justice National Forensic Institute, Istanbul, Turkey \\ ${ }^{5}$ Department of ORL, Susehri State Hospital, Sivas, Turkey
}

\begin{abstract}
Objective: This study aimed to investigate the biomechanical qualities of the perichondrium and cartilage, and to determine the strength of the septal cartilage against bending forces.
\end{abstract}

Study Design: This paper describes an experimental cadaver study.

Materials and methods: The nasal septal cartilages of 14 fresh cadavers (8 hours postmortem) were excised from the cadavers and cut into two strips: one with the perichondrium (group A) and one without perichondrium (group B). A bending test was then performed on the strips.

Results: The deflection of group A strips was larger than the deflection of group B strips. Flexural strength was also larger in group A strips compared to group B strips. The average modulus of elasticity was $122 \%$ higher in group A compared to group B. All conducted tests revealed statistically significant differences between groups.

Conclusion: This study objectively shows that the perichondrium provides the cartilage with a $25 \%$ bending strength.

Key words: septal cartilage, perichondrium, biomechanics, bending test

\section{Introduction}

The nasal septum mucoperichondrium consists of four layers: the mucosal layer; the basal layer, also known as the submucosal-supraepithelial connective tissue; the lamina propria, also known as the subepithelial connective tissue; and the perichondrium, which is the deepest layer ${ }^{(1)}$. There are many studies stating the importance of the perichondrium in preserving the cartilage and providing septum vitality; the perichondrium has protective and nourishing properties for septal cartilage ${ }^{(2-4)}$. The importance of the perichondrium has surfaced through the examination of results of nasal septal surgeries such as septorhinoplasty, septoplasty, and submucosal resection. Many otolaryngology surgeons have been taught to work on the sub- mucoperichondrial level during their education (2).

Biomechanical studies allow exploration of the nose's response to potential external pulling, tension, twisting, or a combination of these impacts, impacts which it may be subjected to in daily life. While the support that the perichondrium provides to the nose is well known, the mechanical effects of the perichondrium on the cartilage remain unclear.

This study aimed to investigate the biomechanical qualities of the perichondrium and the cartilage, and determine the strength that septal cartilage has against bending forces with and without a perichondrium. 


\section{Materials and methods}

Ethical approval was obtained from the Training and Work Council of the Ministry of Justice, Turkey (equivalent to an ethics committee for studies on fresh cadavers).

\section{Tissue preparation}

The nasal septal cartilages of 14 fresh cadavers without nasal septal deviation or any history of nasal trauma (4 females and 10 males; maximum of 6-8 hours postmortem) were completely excised along with the mucosa, while leaving a $0.5 \mathrm{~cm}$ support in the front. The materials were preserved by being wrapped in gauze soaked in $0.9 \% \mathrm{NaCl}$ (in a cold chain) and were transferred to the Yildiz Technical University Metallurgical and Material Engineering Laboratory. Each septum was excised and two equal rectangular strips were formed from the excised tissue. All strips were taken from exactly the same site in each case. The strips were prepared as samples of: perichondrium and cartilage, stripped of the mucosa on both surfaces; and cartilage only, separated from both mucosa and perichondrium on both surfaces under microscopy.

Entry into the subperichondrial and submucosal layers was obtained using a scalpel with a number 15 blade; the tissue was elevated from the septum with a Freer elevator. The thickness of each sample was measured with a digital caliper (Mitutoyo digimatic caliper 500-151U-CD-15C-UK), which had $0.01 \mathrm{~mm}$ sensitivity.

\section{Mechanical testing}

The prepared cartilage strips were subjected to a three-point bending test. Each strip was placed freely on supports that were $10 \mathrm{~mm}$ apart, and the load applicator was located above the center of the strip (Figure 1). Deflection was imposed at a rate of $1 \mathrm{~mm} / \mathrm{s}$, and the load was measured at a resolution of 0.001 $\mathrm{N}$. As there are no described standards for cartilage bending, we referred to the standard for plastic materials ${ }^{(5)}$. The test was carried out on a servo hydraulic bending device (Mares, Tst-sej2T, Turkey) with a $50060.02 \mathrm{~N}$-capacity load cell and $0.1 \mathrm{~mm}$ deflection sensitivity. The results were measured digitally, and the force-deflection curve was plotted automatically (in $\mathrm{mm}$ ). Flexural strength (in MPa) and flexural modulus of elasticity (in $\mathrm{MPa}$ ) values were determined.

Strength (sf) can be defined as the ability of a material to withstand deformation under applied loading without failure. The modulus of elasticity (Ef) was calculated from the slope of the linear portion, which is the elastic region of the flexural stressstrain curve (obtained from the force-deflection curvefor sample dimensions). The stiffer the material, the higher the Ef and the more difficult the material was to deform ${ }^{(6,7)}$.

For the three-point bending test, the specimen is deflected
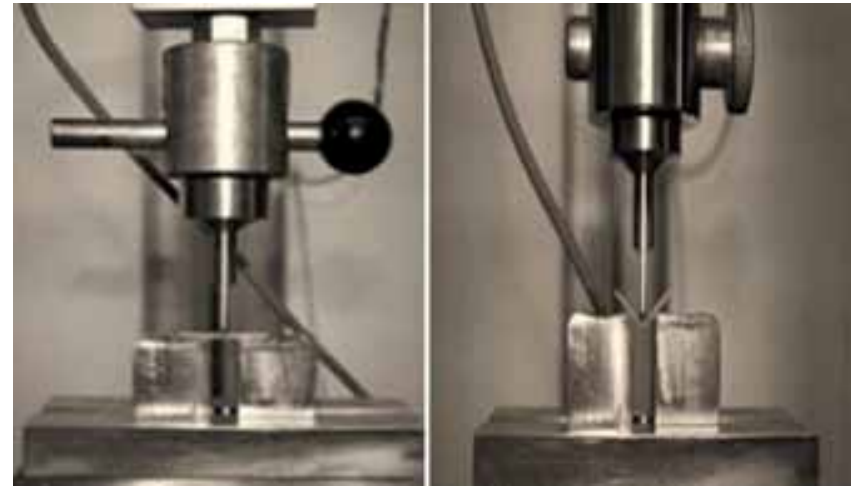

Figure 1. Application of the three-point bending test.

at a constant rate until rupture occurs at the outer surface of the specimen. During this procedure, the force applied to the specimen and the resulting midspan deflection of the specimen is measured. Unless a structural defect exists on the sample, rupture will occur at midspan.

Fracture formation was observed in the experiments. All samples ruptured at their midspans and the force values at rupture were obtained (defined as Fmax). Fractographic analysis was performed on the fractured sections of the bent samples using a scanning electron microscope.

\section{Statistical methods}

SPSS version 16 software was utilized for statistical analysis. Demographic values were reported in terms of averages and standard deviations. The Kolmogorov-Smirnov test revealed that all dependent variables were normally distributed. The data were therefore analyzed using the paired samples t-test; $\mathrm{p}<$ 0.05 was taken as an indication of significance.

\section{Results}

Of the 14 cadavers (from which samples were taken), four were female. The average age of death was $49.92 \pm 15$ years. The average thickness of the samples with the perichondrium intact was $2.10 \pm 0.34 \mathrm{~mm}$, and the average thickness of samples without perichondrium was $1.64 \pm 0.32 \mathrm{~mm}$.

Deflection (S), which is correlated with flexibility, represents the depression distance of the mid-point of the sample from its original position. There was a significant difference $(p=0.0001)$ between the deflection of samples with perichondrium (measured to be $3.80 \pm 0.81 \mathrm{~mm}$ ) and the deflection of samples without perichondrium (2.69 $\pm 0.67 \mathrm{~mm}$; Figure 2$)$.

Analysis of bend strength data also revealed a significant difference between sample groups $(p=0.003)$ : those with a perichondrium were found to be $2.01 \pm 0.97 \mathrm{MPa}$, while those without perichondrium were 1.50 $\pm 0.69 \mathrm{MPa}$ (Figure 3). 


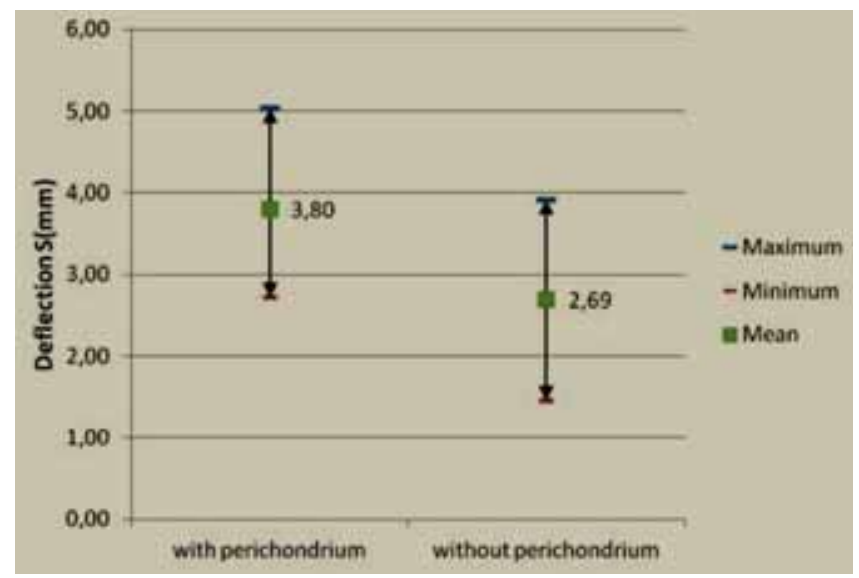

Figure 2. Mean deflection values.

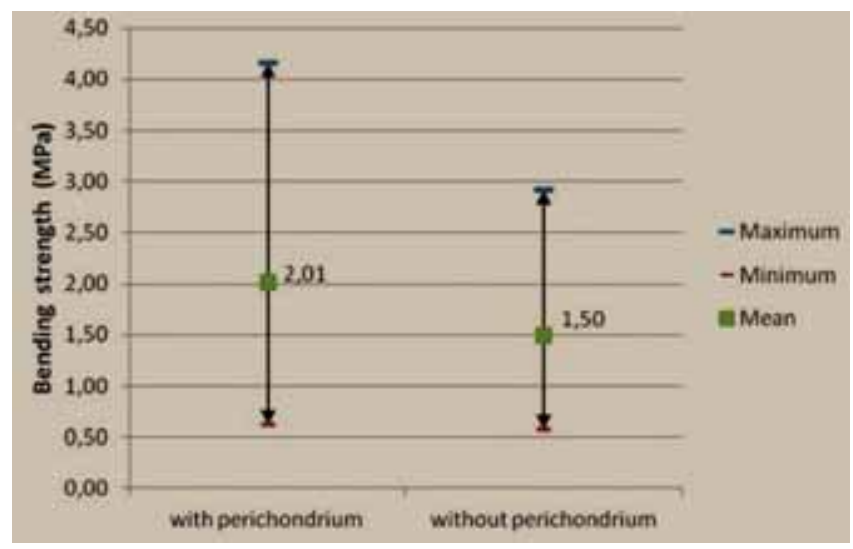

Figure 3. Mean bending strength values.

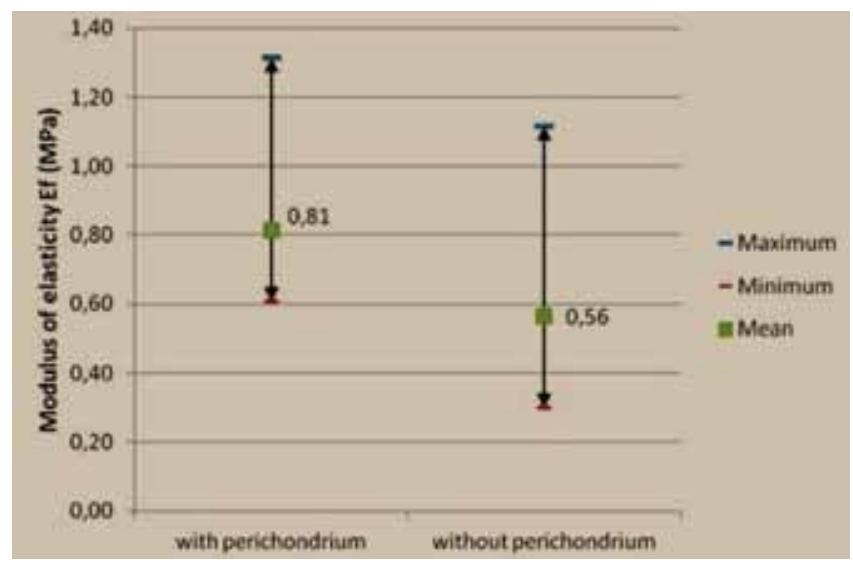

Figure 4. Mean modulus of elasticity values.

In addition, there was a significant difference between groups in terms of the modulus of elasticity $(p=0.0001)$. This was found to be an average of $0.81 \pm 0.18 \mathrm{MPa}$ in samples with perichondrium and $0.66 \pm 0.21 \mathrm{MPa}$ in samples without perichondrium (Figure 4).
In summary, statistically significant differences were found between the groups in the results of all conducted tests. Scanning electron microscopy visuals revealed that the samples with perichondrium had a fractured surface and more plastic deformations; the relative deformation in this group of samples seemed extensive. The perichondrium thickness in this study was measured to be $180 \mu \mathrm{m}$. As samples without perichondrium break in a more brittle manner, the fractographs appear brighter and less deformed. These visuals support the findings for deflection, modulus of elasticity and bend strength.

\section{Discussion}

Many studies have stated the importance of the perichondrium in preserving the cartilage and providing septum vitality ${ }^{(2-4)}$. Its importance has surfaced through examination of the outcome of nasal septal surgeries such as septorhinoplasty, septoplasty, and submucosal resection.

The biggest mistakes in nasal septal surgeries are made during submucosal supraperichondrial dissections. This technique can lead to the resection of the perichondrium and periosteum with the cartilage. Aksoy et al. examined live tissue samples of the septal perichondrium under electron and light microscopy ${ }^{(3)}$. This revealed differences in the sequencing of fibrils between caudal and dorsal septum perichondrium. Their findings led the authors to emphasize the importance that should be given to the perichondrium in septal surgeries and the significance of the structural support that it provides.

If there is inadequate preservation of the perichondrium, the structural support and the contributions that the perichondrium provide to chondrocyte production in the inner layer will be eliminated. Hence, both tissue regeneration and the support mechanism are affected. Kim et al. have stated that tension in the perichondrium and the elimination of the support it provides are the cause of sudden or delayed septal perforation ${ }^{(2)}$. In light of these findings, it has been suggested that perichondrium and cartilage together have a biomechanical role and that these two should be considered jointly as a functional unit. This supports the findings of our study. The same study also compared the perichondrium of septal and lobular cartilage ${ }^{(2)}$. The results showed that the perichondrium of both types of cartilage had a homogenous structure. The authors speculated that morphological changes of the perichondrium's outer and inner layers in the septal, lobular, and triangular cartilage of the nose are caused by functional changes.

The nose is subjected to many external forces, such as compression, tension, bending, and the combination of these impacts. The septum plays an important role in strength-related mechanisms ${ }^{\left({ }^{8}\right)}$. Biomechanical studies allow better understanding of 
these strength mechanisms. Studies on septal cartilage biomechanics generally compare septal cartilage with auricular and costal cartilage, although some have utilized the three-point bending test ${ }^{(9,10-13)}$.

In a study focusing on septal perichondrium biomechanics, Kim et al. separated the septal cartilage of fresh cadavers into mucosa only, perichondrium only, and composite tissue (mucosa and perichondrium), and tested their tension force ${ }^{(2)}$. The findings indicated that the perichondrium is a major soft tissue component that affects biomechanical strength. In a septum where the cartilage has been removed, strength is primarily provided by the perichondrium through the remaining tissue.

Our study strived to form a realistic model without separating the cartilage from the perichondrium. As cartilage tissue is effective in providing tissue support along with soft tissue, it was thought that evaluation of the cartilage and perichondrium, both together and separately, would provide the most valid results.

Our study subjected septal cartilage with and without the perichondrium to three-point bending forces, and measured the support that the perichondrium provides to the cartilage against bending forces. The biomechanical qualities of the cartilage were also tested; this was done objectively, in vitro, using a compression test. The cartilage tissue is a biphasic material that includes solid and liquid phases. The solid phase comprises proteoglycans with a fairly short half-life following death ${ }^{(14)}$. We therefore opted to use fresh cadavers with a maximum of 8 hours since time of death.

The deflection difference between the two groups was $29.21 \%$. This indicates that the septal cartilage is approximately $30 \%$ stronger with perichondrium on either side. The value that best reflects the test results is strength (i.e., the maximum stress that a material can support before failure). Cartilage with perichondrium provided $25 \%$ strength against external impact to which the nose may be subjected. As the modulus of elasticity increases, it becomes more difficult to deform the material. This test suggested that cartilage with perichondrium was $18 \%$ more effective at preserving shape under impact.

Scanning electron microscopy visuals of the samples with perichondrium showed extensive deformation; their fractured surfaces demonstrated that they were subjected to greater plastic deformation. As the samples without perichondrium broke in a more brittle manner, their fractographs were brighter and less deformed. These visuals support the findings of deflection, modulus of elasticity and strength tests.
Damage to the perichondrium is generally avoided during septum surgery based on the belief that the perichondrium provides structural support for the cartilage and helps to nourish it. This study objectively shows that the perichondrium provides the cartilage with a $25 \%$ bending strength. In terms of practical importance, the high elasticity modulus for the samples with perichondrium means that following an external impact to the nose, the septum is $18 \%$ more effective at retaining its previous shape without breaking.

The study has certain limitations. The importance of the perichondrium for cartilage strength in terms of unilateral damage could not be calculated; however, in reality the perichondrium is most frequently damaged unilaterally during surgery. In addition, as this was a cadaver study, which limits the number of samples that can be included, only a small number of samples were used in this study. This meant that differences could not be analyzed statistically on the basis of age and gender. Analysis of a wider series of studies comprising greater numbers of cadavers should be undertaken in the future. Furthermore, the septal cartilage does not exist in isolation but is fixed by the surrounding bone, cartilages and other soft tissues. Therefore, it may not be appropriate to extrapolate from small pieces of individual cartilage to real-life physiological and pathological situations.

In conclusion, this study shows objectively that the perichondrium provides the cartilage with a $25 \%$ bending strength. The deflection and elasticity modulus values of a septum with intact perichondrium are higher than those of cartilage without perichondrium. The findings support the importance of the perichondrium, particularly its role in supporting the cartilage.

\section{Acknowledgement}

None

\section{Authorship contribution}

NST contributed to the data collection and writing of the paper. ZA contributed to the data collection.

OY participated in the writing of the paper.

$A B$ performed the data analysis.

AU performed the data analysis.

FS contributed to the data collection.

HHB participated in the writing of the paper.

EA contributed to the data collection.

YMD helped to write the paper.

\section{Conflict of interest}

We have no financial relationship with the organization that sponsored the research. 


\section{References}

1. Fawcett DW, Jensh RP. Respiratory system. In: Bloom and, Fawcett DW, eds. Concise Histology. New York: Chapman \& Hall, 1997; 230-233.

2. Kim DW, Egan KK, O'Grady K, Toriumi DM. Biomechanical Strength of Human Nasal Septal Lining: Comparison of the Constituent Layers. Laryngoscope. 2005; 115 : 1451-1453.

3. Aksoy F, Yıldırım Y S, Demirhan H, Özturan O, Solakoglu S. Structural characteristics of septal cartilage and mucoperichondrium. J Laryngol Otol. 2012; 126: 38-42.

4. Bleys RLAW, Popko M, De Groot JW, Huizing EH. Histological structure of the nasal cartilages and their perichondrial envelope II. The perichondrial envelope of the septal and lobular cartilage. Rhinology. 2007; 45: 153-157.

5. American Society for Testing and Materials (ASTM) International Standard D79010. Standard Test Methods for Flexural Properties of Unreinforced and Reinforced Plastics and Electrical Insulating Materials.
PA: ASTM International; West Conshohocken; 2010.

6. Huston RL. Principles of Biomaterials. Boca Raton, FL: CRC Press; 2009: 79-111.

7. Park JB. Biomaterials: An Introduction. New York, NY: Plenum Press; 1979: 7-21.

8. Alkan Z, Yigit O, Acioglu E, et al. Tensile characteristics of costal and septal cartilages used as graft materials. Arch Facial Plast Surg. 2011; 13: 322-326.

9. Westreich RW, Courtland HW, Nasser P, Jepsen K, Lawson W. Defining nasal cartilage elasticity: biomechanical testing of the tripod theory based on a cantilevered model. Arch Facial Plast Surg. 2007; 9: 264-270.

10. Granik G, Stein I. Human Ribs: static testing as a promising medical application. J Biomechanics. 1973; 8: 237-240.

11. Stein I, Granic G. Rib structure and bending strength: An autopsy study. Calcif Tissue Res. 1976; 20: 61-73.

12. Sacreste J, Brun-Cassen F, Fayon A.Tarriere C, Got C, Patel A. Proposal for thorax tolerance level in side impacts based on 62 tests performed with cadavers having known bone condition. SAE 1982; 821157

13. Yoganandan N, Pintar FA. Biomechanics of human thoracic ribs. J Biomech Eng. 1998; 120: 100-104.

14. Park JB. Chapter 2, Characterization of Materials. Biomaterials. New York, NY: Plenum Press; 1979:7-21.

H. Huseyin Balikci

Suşehri Devlet Hastanesi

Kulak Burun Bogaz Klinigi

58600 Suşehri, Sivas

Turkey

Tel.: +90-346-311-4008

Fax: +90-346-311-4803

E-mail: balikcient@gmail.com 